\title{
Surgical Treatment of Tarsal Tunnel Syndrome - Case Report
}

EL Maqrout A*, Fekhaoui MR, Boufettal M, Bassir RA, Kharmaz M, Lamrani O, Mahfoud M, Bardouni AEL and Berrada MS

Department of Orthopedic Surgery, University Mohamed V, Morocco

*Corresponding author: Amine EL Maqrout, Department of Orthopedic Surgery, University Mohamed V, Ibn Sina Hospital, Faculty of Medicine of Rabat, Avenue Mohamed Belarbi El Alaoui, Rabat, Morocco

Received: January 04, 2021; Accepted: February 09, 2021; Published: February 16, 2021

\begin{abstract}
The first description of tarsal tunnel syndrome is recent. Koppel in 1960 evoked the after-effects of lesions of the posterior tibial nerve. Keck in 1969 was the first to describe compression of the posterior tibial nerve by the internal annular ligament. It was a young soldier who, after intensive training, had bilateral plantar anesthesia. The opening of the internal annular ligament had allowed a total recovery in 48 hours. Our objective here is to discuss the circumstances of the diagnosis of this syndrome, to analyze its anatomical and pathological causes, to present the types of treatments followed, in the light of the literature.
\end{abstract}

Keywords: Tarsal tunnel; Neurolysis; Surgery

\section{Introduction}

Tarsal tunnel syndrome is a canal syndrome. It concerns the posterior tibial nerve, compressed behind the internal malleolus, in an osteofibrous process consisting of the tibia, the talus and the calcaneus and closed by the deep lamina of the ligament internal annular, extending the aponeurosis of the leg. This tunnel contains, in addition to the posterior tibial nerve, posterior tibial vascular bundle and the tendons of the flexor proper to the big toe, of the common flexor of the toes and of the posterior leg. This syndrome can be caused by trauma, varicose veins, tenosynovitis, compartment tumors or static abnormalities of the hindfoot. In most cases, this syndrome is idiopathic.

\section{Case Presentation}

A young 36-year-old man presented to the consultation for neurological pain of the burn type accompanied by paraesthesia of the inner edge of the ankle and foot. Negative neurological examination (radiculalgia, diabetes and neuropathy) negative static examination of the hindfoot: no palpable mass of the internal retro-malleolar region, irritative neurological syndrome in the form of the sign of percussion, the sign of kinoshita is positive (pain in dorsiflexion associated with prolonged foot eversion 10 seconds). A standard $\mathrm{X}$-ray requested without bone abnormalities. The electromyogram confirms the diagnosis, which shows a slowing down of the speed of sensory conduction.

\section{Surgical Treatment}

It is indicated after at least three months of medical treatment without effect. This medical treatment must combine NSAIDs, physiotherapy, soles, or even plastered immobilization. The operation is carried out under general or locoregional anesthesia. the first is internal retro-malleolar, $6-8 \mathrm{~cm}$ from the top of the malleolus following the path of the posterior tibial nerve to the internal edge of the sole of the foot (Figure 1) the leg fascia is open, continuing on the internal annular ligament and the posterior tibial nerve is located at the upper part of the incision; it is dissected until its termination into internal and external plantar nerves (Figure 2, 3). We will look for any

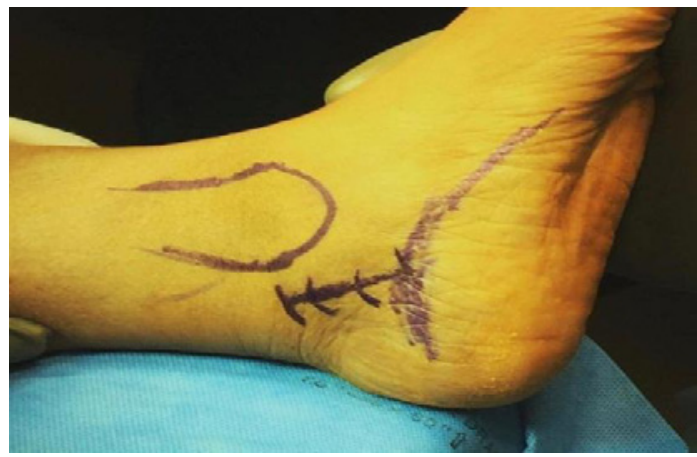

Figure 1: Internal retro-malleolar approach.

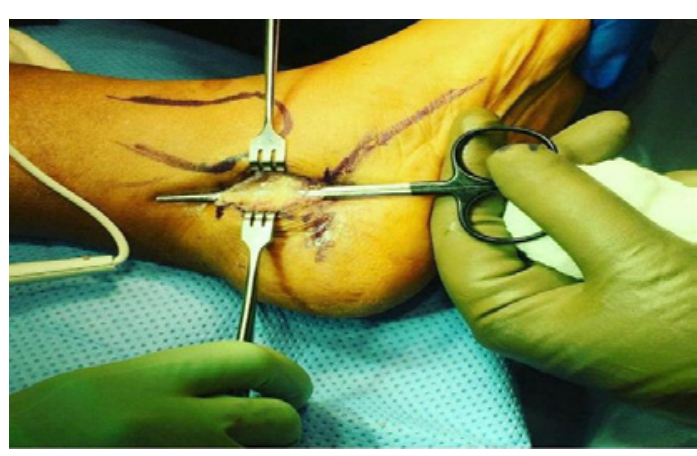

Figure 2: The chisel shows the ligament structure that compresses the tibia nerve behind close to the sole of the foot.

expansive process that may be causing compression. At this level, the deep fascia of the abductor of the big toe is open because it can be a source of compression. The neurolysis practiced is an exo-neurolysis, without inter fascicular dissection on pain of postoperative fibrosis. Careful bipolar coagulation is recommended and hemostasis will be checked by releasing the tourniquet. All the fascias will be left open, only the subcutaneous and skin will be closed. We will do a compression bandage in a posterior splint, then a boot for two weeks
Austin J Orthopade \& Rheumatol - Volume 8 Issue 1 - 202 ISSN: 2472-369X | www.austinpublishinggroup.com EL Maqrout et al. () All rights are reserved
Citation: EL Maqrout A, Fekhaoui MR, Boufettal M, Bassir RA, Kharmaz M, Lamrani O, et al. Surgical Treatment of Tarsal Tunnel Syndrome - Case Report. Austin J Orthopade \& Rheumatol. 2021; 8(1): 1095. 


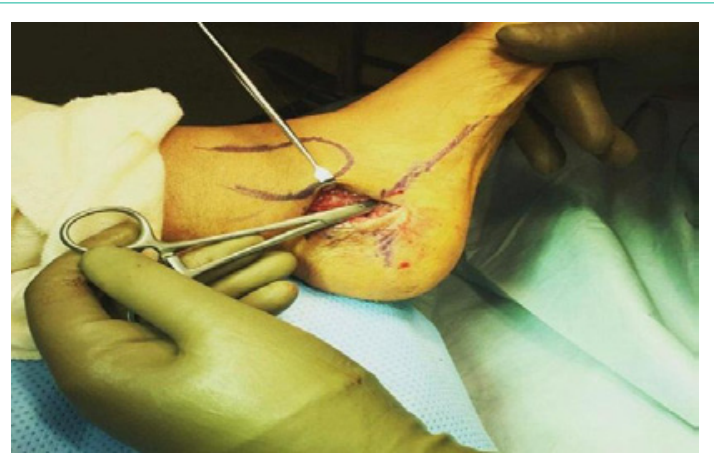

Figure 3: The chisel shows the ligament structure that compresses the tibial nerve behind close to the sole of the foot.

on the fifteenth day after removal of the threads. The support is resumed at one month.

\section{Discussion}

The study of Kaplan and Kern ahan, et al. [1] found a higher frequency of compression of the lateral plantar nerve compared to the median probably linked to its close position, which seems to make it more vulnerable according to them. The best results are obtained, in compressions of the lateral plantar nerve, which corresponded surgically to the complete opening of the internal annular ligament and the complete monitoring of the branches of division of the tibial nerve; this, of course, includes the motor nerve of the fifth toe abductor. This is comparable to the observations of Valtin [2] who recommends a nervous release beyond the division of the nerve and in all cases the deep fascia of the hallux abductor must be incised and insists that this must be the case in any compression of the tibial nerve or one of its branches of division. This recommendation is also taken up by Skalley et al. [3] in their study of 12 cases, similarly in the study of Settanni et al. [4]. Early diagnosis is important because the resulting surgical management guarantees the best results. The study by Kaplan and Kernahan [1] recommends the start of treatment before the onset of muscle atrophy. Takakura et al. [5] estimate from their study that when this delay is more than 10 months the prognosis will be disappointing.
The results of surgical treatment are variable. When a local cause is found, the improvement is rapid. On the other hand, (white) neurolyses cause only half the relief, although the tinel sign disappears and the postoperative EMG normalizes.

Skalley et al. [4] in a study of 12 patients with recurrent tarsal tunnel syndrome, concluded by recommending surgical abstention for patients who received the first release of the tibial nerve and its dividing branches correctly. On the other hand, if severe symptoms and incomplete release of the tibial nerve and its terminal branches persist, he recommends recourse to a new neurolysis to reduce pain, improve activity limitations and reduce pain. Risk of disability. Eberhard and Millesi [6] propose, in their work on 3 recurrences, to transplant the surrounding soft tissues around the nerve in association with a skin graft to increase the dermal envelope if the post-operative fibrosis around the nerve bed has an anchoring to the skin.

\section{Conclusion}

Surgical treatment for tarsal tunnel syndrome should never be a first-line treatment. It depends mainly on a complete release of the tibial nerve and its dividing branches regardless of the compression zone, to obtain a good result. They will be more favorable if the patient is young, without static foot disorder and has severe tarsal tunnel syndrome before the procedure.

\section{References}

1. Kaplan PE, Kernahan WT. Tarsal tunnel syndrome: an electrodiagnostic and surgical correlation. J Bone Joint Surg (Am). 1977; 59: 127-128.

2. Valtin B. Canal syndrome of the posterior tibial nerve and its branches. SOFCOT teaching notebooks. 54: 163-70. French scientific expansion. 2000; 163-170.

3. Settanni FA, Leandro LM, Zuleta JA. Management of tarsal tunnel syndrome: report of seven cases. Arq Neuropsiquiatr. 1994; 52: 530-534.

4. Skalley TC, Schon LC, Hinton RY, Myerson MS. Clinical results following revision tibial nerve release. Foot Ankle Int. 1994; 15: 360-367.

5. Takakura Y, Kidata C, Sugimoto K, Tanaka Y, Tamai S. Tarsal tunnel syndrome. Causes and results of operative treatment. J Bone Joint Surg (Br). 1991; 73: 125-128.

6. Eberhard D, Millesi H. Schmerzsyndrome der $\mathrm{n}$. tibialis am. Ubergang unterschenbel fuss. Wien Klin Wochenschr. 1993; 105: 462-467. 